\title{
Recognition of Affect Conveyed by Text Messaging in Online Communication
}

\author{
Alena Neviarouskaya ${ }^{1}$, Helmut Prendinger $^{2}$, and Mitsuru Ishizuka ${ }^{1}$ \\ ${ }^{1}$ University of Tokyo, Department of Information and Communication Engineering, Japan \\ lena@mi.ci.i.u-tokyo.ac.jp, ishizuka@i.u-tokyo.ac.jp \\ ${ }^{2}$ National Institute of Informatics, Japan \\ helmut@nii.ac.jp
}

\begin{abstract}
In this paper, we address the task of affect recognition from text messaging. In order to sense and interpret emotional information expressed through written language, rule-based affect analysis system employing natural language processing techniques was created. Since the purpose of our work is to improve social interactivity and affective expressiveness of computer-mediated communication, we decided to tailor the system to handle style and specifics of online conversations. Proposed algorithm for affect analysis covers symbolic cue processing, detection and transformation of abbreviations, sentence parsing, and word/phrase/sentence-level analyses. To realize visual reflection of textual affective information, we have designed an avatar displaying emotions, social behaviour, and natural idle movements.
\end{abstract}

Keywords: Affective computing, affective user interface, avatar, emotions, online communication, language parsing and understanding, text analysis.

\section{Introduction and Motivation}

There is a wide perception that the future of human-computer interaction is related to affective computing. The necessity to design intelligent user interfaces and to create rich mediating environments for social interactions is a strong incentive for many researchers to analyze natural language with regard to affective information. Recognition, classification and understanding of opinionated or emotional text are challenging tasks for natural language researchers.

In order to support applications based on language recognition and language production, the linguistic resource for a lexical representation of affective knowledge, WordNet-Affect, was introduced by Strapparava and Valitutti [17]. In [7], authors describe how the structure of the WordNet database might be used to assess affective or emotive meaning. Kim and Hovy [8] developed an automatic algorithm for obtaining opinion-bearing and non-opinion-bearing words, and described a method for detection of sentence-level opinion. An approach to analyzing affect content in free text using fuzzy logic techniques was proposed by Subasic and Huettner [18].

Statistical language modelling techniques have been applied by researchers to learn the characteristics of 'happy' and 'sad' moods indicated in the blog entries [11], and 
to classify online diary posts by mood [9], [12]. However, the main limitations of the "bag-of-words" approach to textual affect classification are neglect of negation constructions and syntactical relations in sentences.

Keyword spotting technique was employed by the emotion recognition system proposed by Olveres et al. [15], and it was used as a method in an approach to multimodal emotion recognition from speech signals and textual content described in [19]. However, a simple word-level analysis model cannot output an appropriate emotional state in cases where affect is expressed by phrases requiring complex phrase/sentencelevel analysis or when a sentence carries affect through underlying meaning. A pure affective keyword spotting technique will fail even with simple sentences like "I saw this movie without interest".

More advanced systems for textual affect recognition, such as the Text-to-Emotion Engine [2] or Empathy Buddy [10], perform sentence-level analysis. Both systems use a small set of emotions, the six "basic" types as defined by Ekman [5]. The parser described in [2] generates emotional output only if an emotional word refers to the person himself/herself and the sentence is in present continuous or present perfect continuous tense. We think that such limitations greatly narrow the potential of textual emotion recognition. As the result, sentences like "Onion pie is disgusting" and "It was the most joyous feeling!" are disregarded by the parser despite the fact that they evidently carry affect. An approach aimed at understanding the underlying semantics of language using large-scale real-world commonsense knowledge is proposed by Liu et al. [10].

Style and level of formalism of written natural language differ greatly depending on situation. In news, reports, scientific papers etc., text is syntactically correct and written in a formal style, while in private correspondence, online messaging, and blogs, text is informal and may include special symbols, emoticons, abbreviations and acronyms.

The weakness of most affect recognition systems integrated with a chat or e-mail browser is that they do not take into account crucial aspects of informal online conversation such as its specific style and evolving language. In order to account for the peculiarity of this medium, and to ensure satisfactory results on real examples, we investigated style, linguistic and interactional features of online conversations (details are given in [14]), and considered them while constructing our Affect Analysis Model.

Social interactions among people play an important role in the establishment of genuine interpersonal relationships and communities. However, computer-mediated communication lacks such signals of face-to-face communication as spoken language, intonation, gaze, facial expressions, gestures, and body language. The main goal of our research is thus to enrich social interactivity and affective expressiveness of online Instant Messaging (IM) communication. Here, a key issue is to support the automation of multiple expressive channels so that the user does not have to worry about visual self-presentation as in standard IM systems, but can focus on the textual content of the conversation. Our approach is based on deep word/ phrase/sentencelevel analyses of affect in text, and the visual reflection of affective states and communicative behaviour through use of a $2 \mathrm{D}$ cartoon-like avatar. 


\section{Basis for Text Categorization}

A fundamental task for any automatic emotion detection system is to first choose the basis for text categorization.

\subsection{Emotion and Communicative Function Categories}

In a face-to-face communication, people prefer to interact with a person who is expressive, because displayed emotion gives the impression that the speaker is significantly more sociable, open and humorous. Interaction in online conversations might be supported by the expressiveness too.

Facial expressions, gestures, body postures and movements have great communicative power [1], [3], [16]. All types of expressive means are dependent on context.

As the purpose of affect recognition in an IM system is to relate text to avatar emotional expressions, emotional categories were confined to those that can be visually expressed. For text categorization, we have decided to use (the relevant) nine emotional states taken from a set of ten emotions defined by Izard [6]: 'anger', 'disgust', 'fear', 'guilt', 'interest', 'joy', 'sadness' ('distress'), 'shame', and 'surprise'.

In our work, we aim at recognition of not only affective information conveyed by textual messages but also communicative functions that can be performed by avatar communicative behaviour ('greeting', 'thanks', 'posing a question', 'congratulation', and 'farewell').

\subsection{Affect Database}

In order to handle abbreviated language and to interpret affective features of emoticons, abbreviations, and words, we created the database using MySQL 5.0 [13].

While accumulating affect database entries, we collected 364 emoticons, both of American and Japanese style (for example, ":">" and " $={ }^{\wedge}{ }^{\wedge}=$ " for 'blushing'), and the 337 most popular acronyms and abbreviations, both emotional and non-emotional (for example, "LOL" for 'laughing out loud', and "4U" - 'for you'). From the source of affective lexicon, WordNet-Affect [17], we have taken 1620 words: adjectives, nouns, verbs, and adverbs. We added not only words that refer directly to emotions, mood, traits, cognitive states, behaviour, attitude, sensations, but also words (especially, verbs) that carry the potential to provoke affective states in humans to our database. Since interjections, such as "alas", "wow", "yay", "ouch", etc. are specific indicators of communicated emotion caused by unexpectedness, a long-awaited joyful event, or pain, they were collected as well. Moreover, we included modifiers (e.g. "very", "extremely", "less", "not", etc.) into our database because they influence the strength of related words and phrases in a sentence.

Emotion categories and intensities were manually assigned to affect-related entries of database by three independent annotators. Intensity values range from 0.0 to 1.0 , and describe the intensity degree of affective states from 'very weak' to 'very strong'. Annotators conformed to our guideline with the description of emotional state gradation within intensity levels. For example, 'cheerful', 'glad', 'happy', 'joyful' and 
'elated' all correspond to the 'joy' emotional state, but to a different degree of intensity. Emoticons and emotional abbreviations were transcribed and related to named affective states, whereby each entry was assigned to only one category (examples are listed in Table 1). The inter-rater agreement was calculated using Fleiss' Kappa statistics. The Kappa coefficients for emoticons and abbreviations are 0.94 and 0.93 , respectively, showing good annotation reliability.

Table 1. Examples of emoticons and abbreviations taken from affect database

\begin{tabular}{cccc}
\hline $\begin{array}{c}\text { Symbolic } \\
\text { representation }\end{array}$ & Meaning & Category & Intensity \\
\hline$:-)$ & happy & Joy & 0.6 \\
$:-\mathrm{O}$ & surprise & Surprise & 0.8 \\
$:-\mathrm{S}$ & worried & Fear & 0.4 \\
$/\left(\wedge \mathrm{O}^{\wedge}\right) /$ & very excited & Joy & 1.0 \\
$(\sim-\sim)$ & grumpy & Anger & 0.3 \\
$\mathrm{~m}(.-.) \mathrm{m}$ & bowing, thanks & Thanks & - \\
$\mathrm{JK}$ & just kidding & Joy & 0.3 \\
$4 \mathrm{gv}$ & forgive & Guilt & 0.6 \\
$\mathrm{PPL}$ & people & - & - \\
\hline
\end{tabular}

Considering the fact that some affective words may express more than one emotional state, annotators related those words to more than one category. For instance, 'anger' and 'sadness' emotions are involved in the annotation of word "frustrated" with intensities 0.2 and 0.7 , respectively (Table 2 ).

Table 2. Examples of words taken from affect database

\begin{tabular}{cccc}
\hline Affective word & Part of speech & Category & Intensity \\
\hline cheerfulness & noun & Joy & 0.3 \\
amazing & adjective & Surprise & 1.0 \\
frustrated & adjective & Anger & 0.2 \\
& & Sadness & 0.7 \\
dislike & verb & Disgust & 0.4 \\
hopefully & adverb & Interest & 0.2 \\
& & Joy & 0.3 \\
\hline
\end{tabular}

Variance of data from the mean was taken into consideration in order to eliminate errors in resulting intensity estimation due to subjective judgements. If the variance was not exceeding the threshold in 0.027 , the resulting intensity was measured as the average of intensities given by three annotators. Otherwise, the intensity value responsible for exceeding the threshold was removed, and only the remaining values were taken into account.

As for the modifiers, coefficients for intensity degree strengthening or weakening were given (e.g. 1.4 for "very"). 


\section{Affect Analysis Model}

The algorithm for analysis of affect in text consists of five stages:

1. symbolic cue analysis;

2. syntactical structure analysis;

3. word-level analysis;

4. phrase-level analysis;

5. sentence-level analysis.

The working flow of the Affect Analysis Model is presented in Fig. 1.

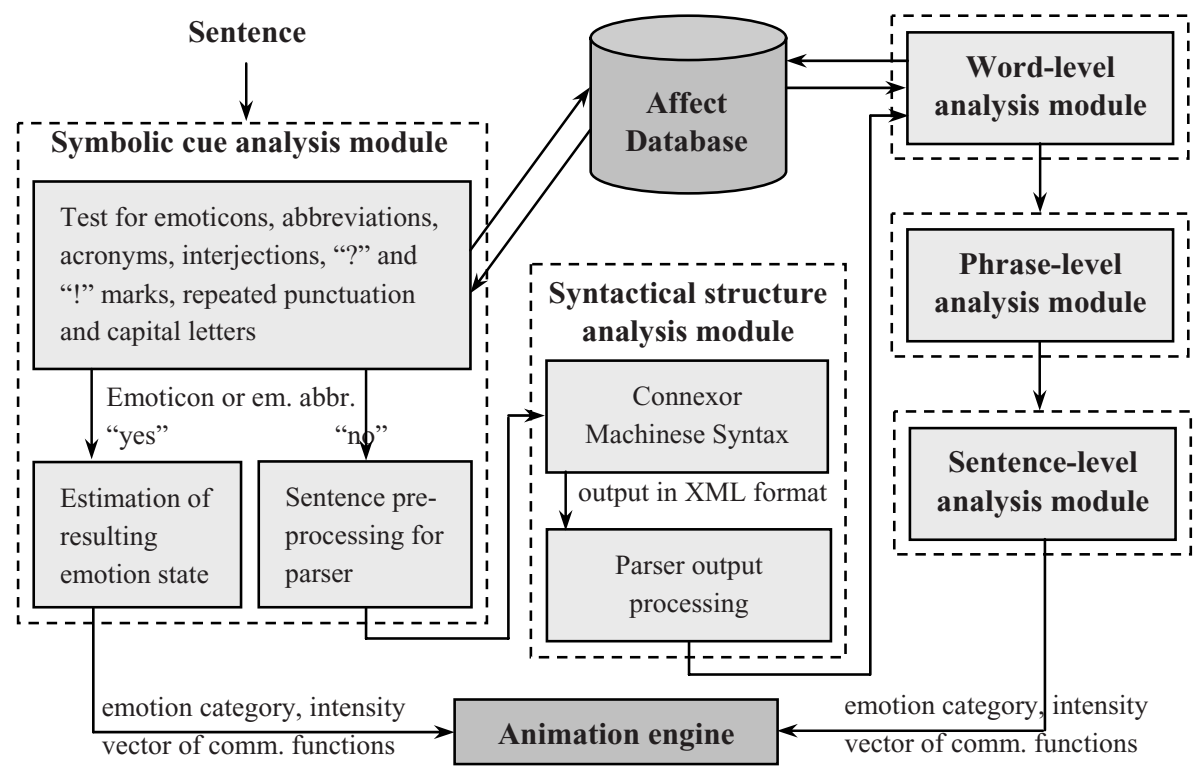

Fig. 1. Working flow of the Affect Analysis Model

\subsection{Symbolic Cue Analysis Module}

In the first stage, the sentence is tested for occurrences of emoticons, abbreviations, acronyms, interjections, "?" and "!" marks, repeated punctuation and capital letters.

First of all, punctuation marks of a sentence are delimited from words in order to disambiguate sentence punctuation marks from those belonging to emoticons. The "!" mark, repeated punctuation and capital letters are considered as an emphasis of the communicated emotion.

If there is an emoticon or abbreviation related to an emotional state, no further analysis of affect in text is performed based on the assumption that the emoticon (or abbreviation) dominates the affective meaning of the entire (simple or compound) sentence. It is known that people type emoticons and emotional abbreviations to show actual feeling, or to avoid misleading the other participants, for instance, after irony or 
joke. On the other hand, if there are multiple emoticons or emotion-relevant abbreviations in the sentence, we determine the prevailing (or dominant) emotion based on the following two rules: (1) when emotion categories of the detected emoticons (or abbreviations) are the same, the higher intensity value is taken for this emotion; (2) when they are different (e.g. 'sad': 0.5 and 'joy': 0.2 ), the category (and intensity) of the emoticon occurring last is dominant.

Regarding the visualisation by the avatar, when both emotional state and communicative function category appear in a sentence (for example, 'joy' and 'thanks'), two animations are sequentially displayed.

As interjections are added to text to reflect an author's feelings, like in the sentences "Oh no, I forgot that the exam was today!" and "But anyways, yay!", they are analysed as well.

In case of an interrogative sentence, we process it further at subsequent stages in order to identify whether the question expresses strong emotion or not. While some researchers ignore such sentences at all, we believe that questions, like "Why do you irritate me so greatly?" may carry emotional content.

If there are no emotion-relevant emoticons or abbreviations in a sentence, we prepare the sentence for parser processing: emoticons and abbreviations standing for communicative function categories are excluded from the sentence, and nonemotional abbreviations and acronyms are replaced by their proper transcriptions found in the database. In such a way, the problem of correct processing of abbreviated text by syntactical parser is settled.

\subsection{Syntactical Structure Analysis Module}

The second stage is devoted to syntactical structure analysis. The used deep syntactical parser, Connexor Machinese Syntax [4], returns exhaustive information for analysed sentences, including word base forms, parts of speech, dependency functions, syntactic function tags, and morphological tags. From the parser output in XML style, we can read off the characteristics of each token and the relations between them in a sentence (e.g. subject, verb, object, and their attributes).

\subsection{Word-Level Analysis Module}

After handling the result from the previous analysis stage, the system transfers the data to the third stage, word-level analysis.

For each word found in the database, either the communicative function category is taken as a feature or the affective features of a word are represented as a vector of emotional state intensities e $=$ [anger, disgust, sadness, fear, guilt, interest, joy, shame, surprise] (e.g. e $=[0.2,0,0.7,0,0,0,0,0,0]$ for word "frustrated").

In the case of a modifier, the system identifies its coefficient.

Since the database contains words only in their dictionary form, one important system function on this stage is to increase the intensity of the emotional vector of an adjective if it is in comparative or superlative form. Currently, the intensity of an adjective is multiplied by the values 1.2 or 1.4 , depending on its form. 


\subsection{Phrase-Level Analysis Module}

In the fourth stage, phrase-level analysis is performed. The purpose of this stage is to detect emotions involved in phrases. Words in a sentence are interrelated and, hence, each of them can influence the overall meaning and sentiment of a statement.

We have defined general types of phrases, and rules for processing them with regard to affective content:

- adjectival phrase ("extremely sad"): modify the vector of adjective;

- noun phrase ("wonderful peace"): output vector with the maximum intensity within each corresponding emotional state in analysing vectors (for instance, e $1=[0 . .0 .7 .$. and e2=[0.3..0.5..] yield e3=[0.3..0.7...]);

- verb plus noun phrase: if verb and noun phrase have opposite valences ("break favourite vase", "enjoy bad weather"), consider vector of verb as dominant; if valences are the same, output vector with maximum intensities in corresponding emotional states for positive ("like honey"), and output null vector for negative;

- verb plus adjective phrase ("is very kind", "feel bad"): output vector of adjective phrase.

The rules for modifiers that influence the emotional vectors of related words are as follows:

- intensifiers multiply or decrease emotional intensity values;

- negation modifiers such as "no" or "not", and connector "neither...nor" cancel (set to zero) vectors of the related words, i.e. "neutralize the emotional content";

- prepositions such as "without", "except", "against", "despite" cancel vectors of related words.

We think that negation constructions do not reverse emotional meaning of words from positive to negative or vice versa. For example, "not splendid" is not necessarily reverse of "splendid".

Statements with words like "think", "believe", "sure", "know" and with modal operators such as "can", "may", "need" etc. are not considered by our system because they express a modal attitude towards the proposition. Conditional clause phrases beginning with "if", "when", "whenever", "after", "before” are disregarded as well.

\subsection{Sentence-Level Analysis Module}

In the fifth and final stage, the overall affect of a sentence and its resulting intensity degree are estimated. The emotional vector of a simple sentence (or of a clause) is generated from emotional categories and their intensities resulting from phrase-level analysis.

It is important to note that the developed system enables the differentiation of the strength of the resulting emotion depending on the tense of a sentence and availability of first person pronouns. We introduce this idea based on psychological literature.

As Paul Ekman states, "sometimes when people give an account of an emotional experience they unexpectedly begin to re-experience the emotion" [5]. "Genuine" 
emotion expressions display that emotion is now felt, whereas so-called "referential" expressions occur most often when people talk about past or future emotional experiences. Therefore, we assume that the strength of emotions conveyed by text depends on tense.

As to first person pronouns, people tend to use them to underline the strength of an emotion. For example, emotion conveyed through sentence like "I am charmed by cherry flowers of Japan" is stronger than in case of "Cherry flowers of Japan are charming".

According to our proposal, the emotional vector of a simple sentence (or of a clause) is multiplied by the corresponding empirically determined coefficient of intensity correction (Table 3 ).

Table 3. Coefficients of intensity correction

\begin{tabular}{ccc}
\hline \multirow{2}{*}{ Tense } & \multicolumn{2}{c}{ First person pronouns } \\
& yes & no \\
\hline present & 1 & 0.8 \\
past & 0.8 & 0.4 \\
future & 0.4 & 0 \\
\hline
\end{tabular}

For compound sentences, we defined two rules:

- with coordinate connectors "and" and "so" (e.g. "Exotic birds in the park were amazing, so we took nice pictures."): output the vector with the maximum intensity within each corresponding emotional state in the resulting vectors of both clauses;

- with coordinate connector "but" (e.g. "Canada is a rich country, but still it has many poor people."): the resulting vector of a clause following after the connector is dominant.

After the dominant emotion of the sentence is determined, the relevant parameters are sent to the animation engine.

\section{Visualization of Affect}

We created an emotively expressive avatar for visual reflection of textual affective information. Animation engine of the developed system is responsible for the display of animations in an appropriate sequence, and for the decision on their duration (depending on sentence length). The strength of the displayed emotion is directly related to the intensity of the emotion derived from the text message.

To achieve believable emotion visualization, the avatar can display various emotions, behaviour associated with communicative functions, and idle states giving a sense of "liveliness".

Examples of 'greeting' and 'surprise' expressions are shown in Fig. 2. 

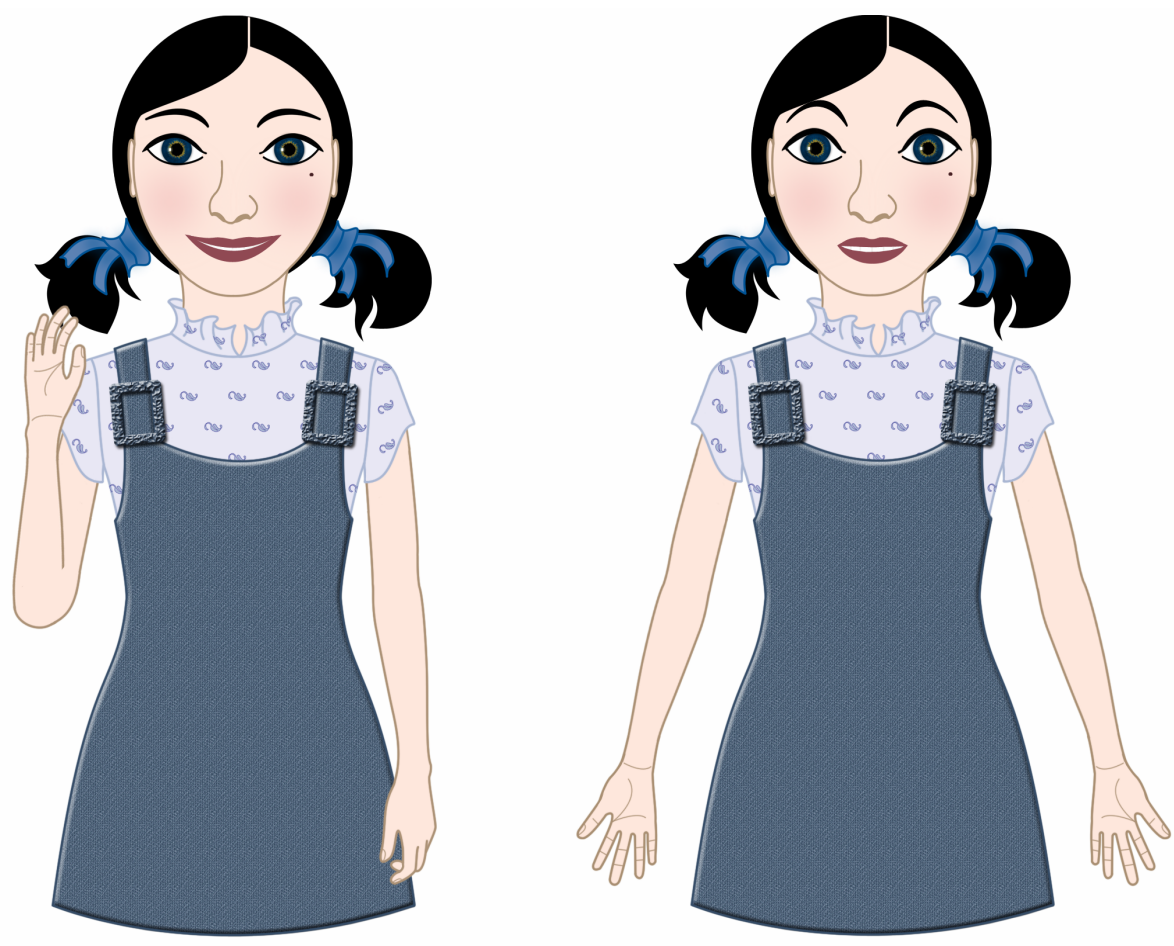

Fig. 2. 'Greeting' and 'surprise' expressions

\section{Conclusion}

This paper has introduced a rule-based syntactical approach to affect recognition from text messaging. Typically, researchers in this field deal with grammatically and syntactically correct textual input. By contrast, our analysis of affect is inspired by the evolving language as seen in online conversation. The purpose of our work is to improve expressiveness and interactivity of computer-mediated communication. For textual input processing, the proposed analysis model takes into consideration features of IM conversation. Affect in text is classified into nine emotion categories, and information that can be displayed by avatar gestures as communicative behaviour is identified. The strength of a displayed emotional state depends on emotional vectors of words, relations among them, tense of sentence and availability of first person pronouns. A designed graphical representation of a user, avatar, performs various expressive patterns, contributing thus to rich interactivity.

Acknowledgments. We would like to express our gratitude to Dzmitry Tsetserukou and Shaikh Mostafa Al Masum who have contributed to annotations of affect database entries for their efforts and time. 


\section{References}

1. Allwood, J.: Bodily Communication Dimensions of Expression and Content. In: Multimodality in Language and Speech Systems, pp. 7-26. Kluwer Academic Publishers, Netherlands (2002)

2. Boucouvalas, A.C.: Real Time Text-to-Emotion Engine for Expressive Internet Communications. In: Being There: Concepts, effects and measurement of user presence in synthetic environments, pp. 306-318. IOS Press, Amsterdam (2003)

3. Cassell, J.: More than Just Another Pretty Face: Embodied Conversational Interface Agents. Communications of the ACM 43(4), 70-78 (2000)

4. Connexor Oy. http://www.connexor.com/

5. Ekman, P.: Facial Expression and Emotion. American Psychologist 48(4), 384-392 (1993)

6. Izard, C.E.: Human emotions. Plenum Press, New York, NY (1977)

7. Kamps, J., Marx, M.: Words with attitude. In: Proceedings of the 14th BelgianNetherlands Conference on Artificial Intelligence, BNAIC'02, pp. 449-450 (2002)

8. Kim, S.-M., Hovy, E.: Automatic Detection of Opinion Bearing Words and Sentences. In: Dale, R., Wong, K.-F., Su, J., Kwong, O.Y. (eds.) IJCNLP 2005. LNCS (LNAI), vol. 3651, Springer, Heidelberg (2005)

9. Leshed, G., Kaye, J.: Understanding How Bloggers Feel: Recognizing Affect in Blog Posts. In: Extended Abstracts of CHI'06, pp. 1019-1024 (2006)

10. Liu, H., Lieberman, H., Selker, T.: A Model of Textual Affect Sensing using Real-World Knowledge. In: Proceedings of IUI'03, pp. 125-132 (2003)

11. Mihalcea, R., Liu, H.: A Corpus-based Approach to Finding Happiness. In: Proceedings of the AAAI Spring Symposium on Computational Approaches to Weblogs (2006)

12. Mishne, G.: Experiments with Mood Classification in Blog Posts. In: Proceedings of the First Workshop on Stylistic Analysis of Text for Information Access (2005)

13. MySQL 5.0. http://www.mysql.com/

14. Neviarouskaya, A., Prendinger, H., Ishizuka, M.: Analysis of Affect Expressed through the Evolving Language of Online Communication. In: Proceedings of IUI'07, pp. 278-281. ACM Press, New York (2007)

15. Olveres, J., Billinghurst, M., Savage, J., Holden, A.: Intelligent, Expressive Avatars. In: Proceedings of WECC'98, pp. 47-55 (1998)

16. Poggi, I., Pelachaud, C.: Performative Faces. Speech Communication 26, 5-21 (1998)

17. Strapparava, C., Valitutti, A.: WordNet-Affect: an Affective Extension of WordNet. In: Proceedings of LREC'04, pp. 1083-1086 (2004)

18. Subasic, P., Huettner, A.: Affect Analysis of Text Using Fuzzy Semantic Typing. In: IEEE Transactions on Fuzzy Systems, vol. 9(4) (2001)

19. Chuang, Z.-J., Wu, C.-H.: Multi-Modal Emotion Recognition from Speech and Text. Computational Linguistic and Chinese Language Processing 9(2), 45-62 (2004) 